\title{
Phase Separation Characteristics through Vertical Y Junction preceded by Elbow Tube
}

\author{
Kosuke Miyawaki ${ }^{1}$, Yoji Onaka ${ }^{1}$ \\ ${ }^{1}$ Mitsubishi Electric Corporation \\ 8-1-1, Tsukaguchi-honmachi, Amagasaki-shi, Hyogo, Japan \\ Miyawaki.Kosuke@da.MitsubishiElectric.co.jp; Onaka.Yoji@db.MitsubishiElectric.co.jp
}

\begin{abstract}
The distribution of two phase refrigerant flow (R32) in the Y junction after a horizontal to vertical $90^{\circ}$ elbow tube are discussed through experimental and analytical approaches. In the experiment, the dependency of the distribution on structural parameters such as the orientation angle of elbow tube as well as operating conditions such as inlet flow rate and quality are evaluated. In addition, the results are analyzed with the reported perceptions for air-water flow. The dependence of R32 distribution in the Y junction on the mass flow ratio of the outlet channels was found to be similar to that of air-water flow. On the other hand, the characteristic dependency on the orientation angle reported for air-water flow was not observed in this study.
\end{abstract}

Keywords: Refrigerant, Two Phase Flow, Distributor, Elbow, Distribution Measurement

\section{Introduction}

Multiphase flow are widely used in industrial facilities and products, especially in HVAC systems, nuclear reactors and oil fields. Many researchers have contributed their efforts to reveal the mechanism of liquid-gas two phase flow over various operating conditions and structural parameters. However, due to the complicated interaction between gas phase and liquid phase depending on refrigerant and flow regimes, further studies are required for comprehensive understanding.

The aim of this study is to evaluate the distribution characteristics of refrigerant flow (R32) in Y junction preceded by elbow tube under various structural and operating conditions. The Y junction, which is a widely used 3 way tube, are often connected to downstream of elbow tubes in products. The designing parameters such as tube sizes and orientation angles are often restricted by product volume, and therefore understanding of governing factor for the distribution in Y junction with elbow tube is important to achieve an improvement in performance and downsizing of products at the same time.

In this study, the two phase flow characteristics from a bend tube to a $\mathrm{Y}$ junction were experimentally investigated using a working fluid of R32 refrigerant, which is widely used hydrofluorocarbon (HFC) refrigerant in HVAC system. In previous studies, many researchers have conducted experimental and simulation approaches for air-water two phase flow and some have reported for HFC refrigerant flow. Aziz and Miyara et al. [1] reported that the positioning of the Y junction has a significant impact on the air-water phase distribution. Azzopardi et al [2] and Yoshimura et al [3] discussed the distribution characteristic on vertical impacting $\mathrm{T}$ junction with the elbow tube. Billiet et al [4] concluded that R32 distribution in horizontal $\mathrm{T}$ junction have some correlation with air-water flow from their experiment. However, according to the author's knowledge, few reports have discussed the split direction of HFC refrigerant through a Y junction with a bend tube. In this report, the distribution experiment for the R32 refrigerant split flow in a Y junction with a bending tube are conducted and the results are comparatively analyzed with earlier studies.

\section{Experiment}

\subsection{Experimental Apparatus}

Figure 1 shows the experimental apparatus to evaluate the phase separation characteristic. The apparatus consists of the general heat pump system and the measurement circuit with a test section. The heat pump cycle in the experiment is as follows. First, the conditions of two phase flow such as mass flow rate $M$ in, pressure $P$ in and quality $x_{\text {in }}$ are controlled at the heat pump system. Then, the refrigerant are supplied to the test section and split into two flow channels at the Y junction, which one outlet channel reaches the downstream junction while the other are connected to the measurement circuit. The measurement circuit consists of a heat exchanger and a thermocouple followed by a fluid meter and an adjust valve, which 
enables to measure and control heating capacity $Q$, superheat degree $T_{\mathrm{sh}}$ and the mass flow rate of the superheated refrigerant flow $M_{1}$. Finally, the separated flow channels join at the downstream junction and return to the heat pump system. The quality of a split channel are evaluated by following equations.

$$
\begin{gathered}
M_{L, 1}=\frac{Q-\left.M_{1} \Delta H\right|_{T_{s h}}}{\left.\Delta H_{s a t}\right|_{P=P_{i n}}} \\
x_{1}=1-\frac{M_{L, 1}}{M_{1}}
\end{gathered}
$$

Figure 2 and table 1 describes the schematic image of the test section and structural parameters, respectively. In this experiment, the outlet quality were measured over several orientation angle $\theta\left(0,90180^{\circ}\right)$ of the elbow tube. The two phase refrigerant flows the test section as follows. First, the refrigerant flows horizontally from the inlet and turn at the elbow tube to vertical flow. Next, the vertical flow runs up after certain run up distance and reaches the Y junction. Finally, the phase separation occurs at the Y junction and separated into two different outlet channels.

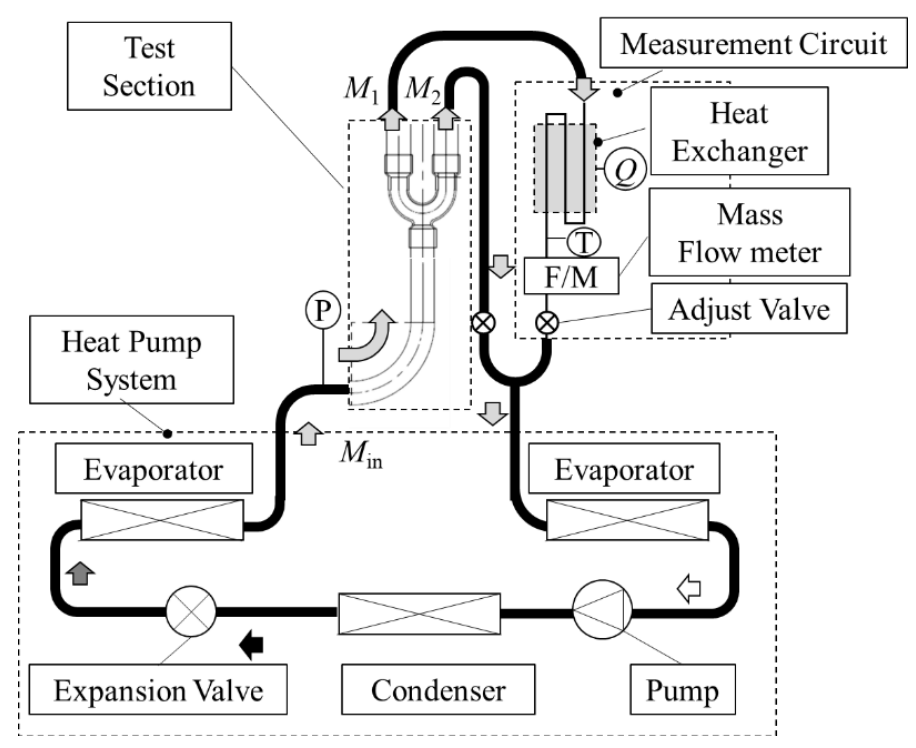

Fig. 1: Experimental Apparatus. 


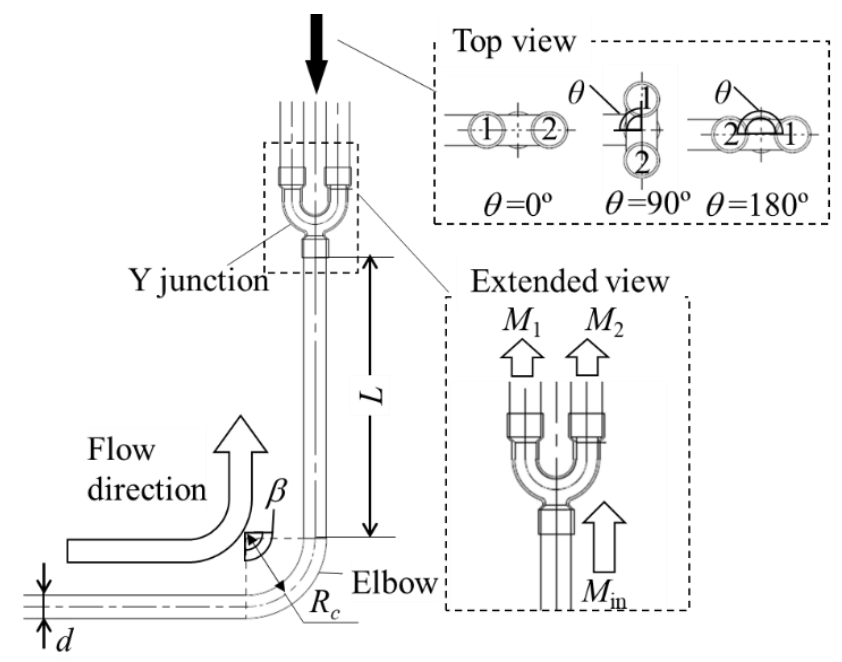

Fig. 2: Schematic image of test section.

Table 1: Structural parameters of test section.

\begin{tabular}{c|c}
\hline Tube Diameter $d[\mathrm{~mm}]$ & 12 \\
\hline Bending Radius $R_{\mathrm{c}}[\mathrm{mm}]$ & 38.1 \\
\hline Bending angle $\left.\beta{ }^{\circ}\right]$ & 90 \\
\hline Run Up Distance $L[\mathrm{~mm}]$ & 140 \\
\hline Orientation Angle $\left.\theta{ }^{\circ}\right]$ & $0, \quad 90,180$ \\
\hline
\end{tabular}

\subsection{Experimental Conditions}

The experimental conditions in this study are listed in Table 2 . The conditions are set with several inlet conditions such as mass flux and quality. Referring to the regime map by Titel et al [5], the flow regimes in this study are considered to be annular flow (A, B, C), and transition of churn to annular flow (D). The phase separation are evaluated at various mass flow ratio at outlet tubes ranged from 30:70 to 70:30.

Table 2: Operating conditions.

\begin{tabular}{c|c|c|c|c}
\hline Condition & A & B & C & D \\
\hline \hline Inlet mass flux $G r_{\text {in }}\left[\mathrm{kg} / \mathrm{m}^{2} \mathrm{~s}\right]$ & \multicolumn{2}{|c|}{1940} & 260 & $45-90$ \\
\hline Inlet quality $x_{\text {in }}[-]$ & 0.23 & 0.60 & 0.23 & $0.05-0.80$ \\
\hline Flow regime & \multicolumn{3}{|c|}{ annular } & churn-annular \\
\hline Mass flow ratio $M_{1} / M_{\text {in }}[\%]$ & \multicolumn{3}{|c|}{$30 \sim 70$} & 50 \\
\hline
\end{tabular}

\section{Result and Discussion}

\subsection{Dependency on the working fluid}

The distribution at $\mathrm{Y}$ junction with elbow tube oriented at 90 degree angles to split direction was compared between experimental data of R32 flow and the prediction of air-water flow. Figure 3 shows the results for the deviation of outlet quality from inlet quality over different mass flow ratio in annular flow condition compared with the prediction for air-water flow proposed by Azzopardi et al. [2]. The positive values on the vertical axis represents gas rich flow, while the negative 
values represents liquid rich flow. In the figure, it could be seen that the phase separation characteristic between mass flow ratios of 30:70 to 70:30 shows good correlation within quality of 0.02.

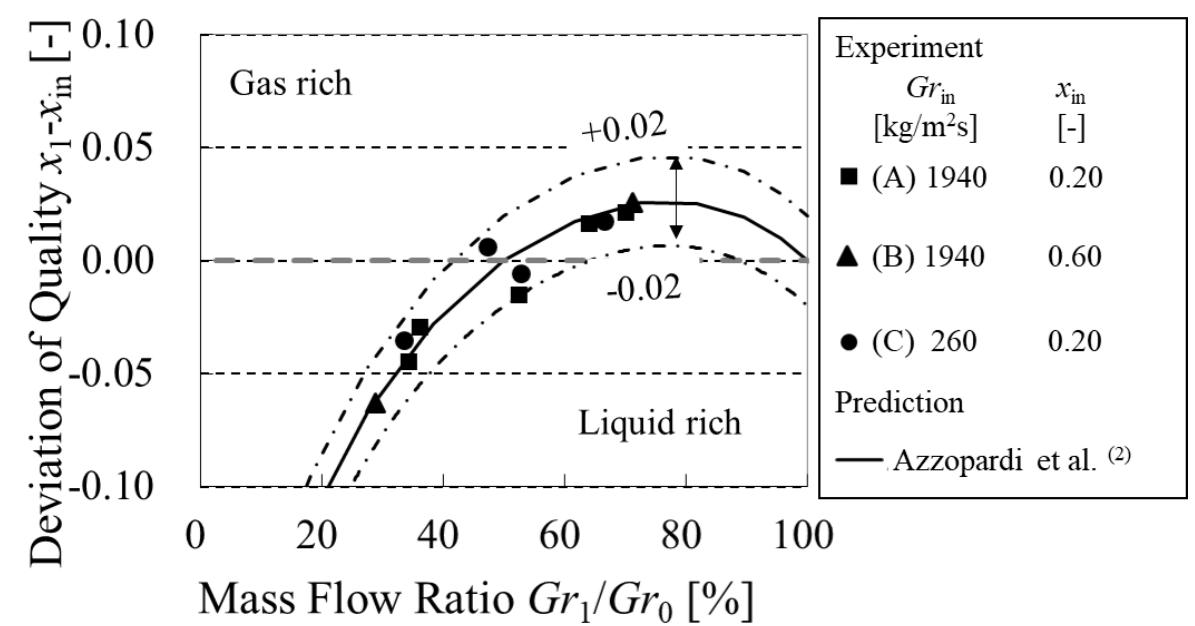

Fig. 3: Comparison of quality characteristic between experimental result for R32 and prediction for air-water for annular flow $\left(\theta=90^{\circ}\right)$.

\subsection{Effect of turning flow at elbow tube}

The impact of a turning flow at the elbow tube on the distribution was evaluated at the $\mathrm{Y}$ junction with the elbow tube oriented at 0 and 180 degree angles to the split direction under mass flow ratio of 50:50. The results for condition $\mathrm{C}$ are shown in Fig. 4. A liquid rich flow was detected at the outlet channel on the outside of the bend, while the flow through the pipe on the inside of the bend was gas rich. The results in various conditions are shown in Fig. 5 indicated by three different markers regarding the quality of the outlet flow on the outside of the bend: filled-, unfilled- and hatched-marker for liquid rich $\left[\left(x_{1}-x_{\text {in }}\right) / x_{\text {in }}<-0.05\right]$, gas rich $\left[\left(x_{1}-x_{\text {in }}\right) / x_{\text {in }}>0.05\right]$ and intermediate $\left[-0.05 \leq\left(x_{1}-x_{\text {in }}\right) / x_{\text {in }} \leq 0.05\right]$ flow, respectively. More liquid tends to flow the outside of the bend in annular flow region, while relatively uniform phase separation was observed in churn flow region. Although Azzopardi et al [2] reported more liquid was taken inside of the bend for air-water flow, the quality inversion rarely occurred in this study. These phenomena can be explained from earlier studies from Gardner and Neller [6] and Wallis [7]. Where the former report discuss the behaviour of air-water flow in bend tubes and the later report describes the flooding condition in vertical tubes.

According to Gardner and Neller [6], the shift in the radial distribution of air-water flows in a bend can be expressed by the competition between the centrifugal force and the gravity using the following modified Froude number

$$
F r_{\beta}=\frac{v_{m}{ }^{2}}{g R_{C} \sin \beta}
$$

In their discussion, more water are taken to the outside of the bend where the Froude number is larger than 1, and more air moves to the outside where the value decreases through unity. This equation explains the results in this study that more liquid phase was distributed to the outlet on the outside of the bend for the higher mass flux conditions, while almost even distribution was found at the lower mass flux conditions.

To discuss the relatively uniform distribution in low mass flux condition, the flooding in upward flow after the bend was evaluated according to the study by Wallis [7], which critical point of a flooding condition is described by following flooding number $C$.

$$
C=j_{L}^{* 0.5}+j_{G}^{* 0.5}
$$




$$
\begin{aligned}
& j_{L}^{*}=j_{L}\left\{\frac{\rho_{L}}{g d\left(\rho_{L}-\rho_{G}\right)}\right\}^{0.5} \\
& j_{G}^{*}=j_{G}\left\{\frac{\rho_{G}}{g d\left(\rho_{L}-\rho_{G}\right)}\right\}^{0.5}
\end{aligned}
$$

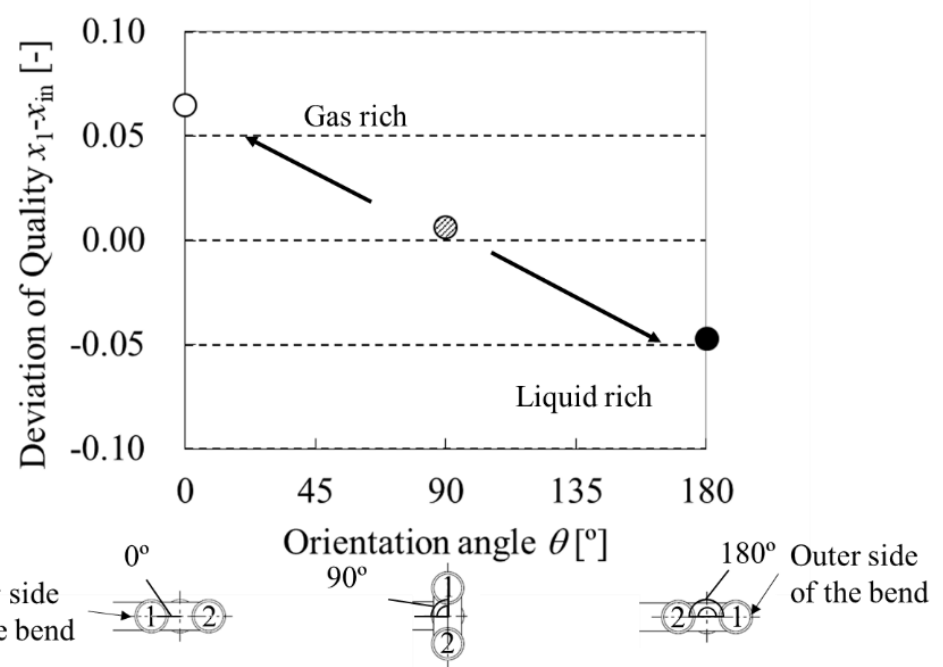

Fig. 4: The deviation of quality under different orientation angle (For condition C, mass flow ratio 50\%).

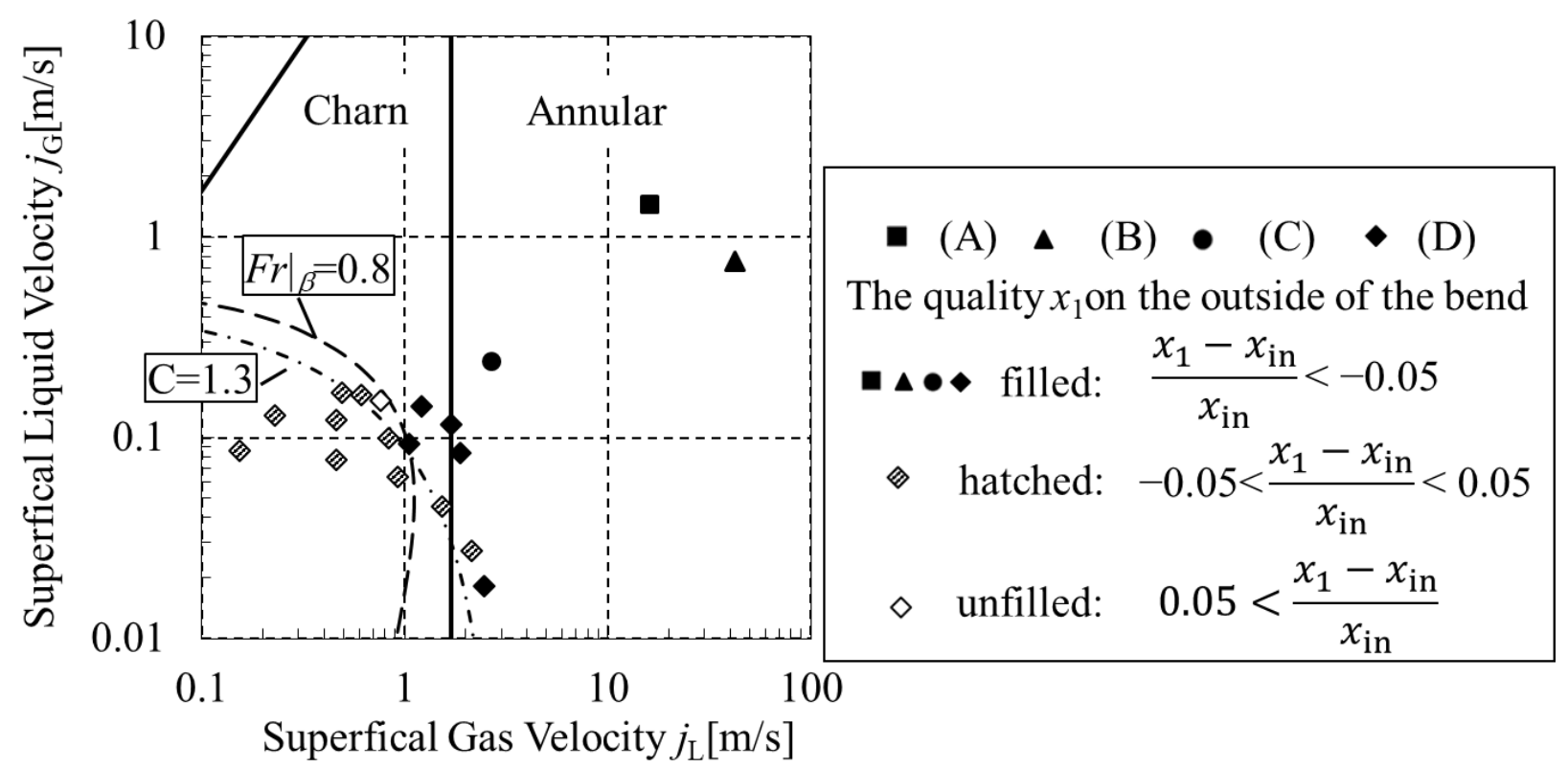

Fig. 5: Experimental data points on Taitel's flow regime map [5] (For mass flow ratio 50\%).

The flooding in a vertical upward flow, which leads more liquid film to drop downward, are considered to decay the radial phase separation in the turning flow, and therefore resulted in small difference of quality in both side of the Y junction.

Based on discussion above, the critical point of Froude number and flooding number was assumed to be 0.8 and 1.3 from this experiment, respectively. However, the value is considered to differ by the working fluid, as well as designing parameters such as run up distance $L$, bending angle $\beta$ and flow resistance of downstream channels, as those factors are 
assumed to affect the centrifugal force and the decay of the phase inhomogeneity, and therefore further inspection is required for comprehensive understanding.

\section{Conclusion}

The phase separation characteristic of R32 at a vertical Y junction preceded by an elbow tube at several orientation angles to split directions was evaluated experimentally and compared with literature perception. The conclusions are as follows.

1. The distribution characteristic at the orientation angle of 90 degree showed similar dependency on mass flow ratios between from 30:70 to 70:30 with reported model for air-water flow within quality of 0.02.

2. The distribution characteristic at orientation angles of 0 and 180 degree suggested that more liquid phase are taken to outside of the turning flow mainly in annular flow condition, while most of the churn flow showed relatively uniform distribution. The modified Froude number of 0.8 and flooding number of 1.3 was proposed as the transition point of the characteristic.

\section{Nomenclature}

$\begin{array}{cll}\text { Latin } & & \\ C & {[-]} & \text { Flooding number } \\ d & {[\mathrm{~m}]} & \text { Inner diameter } \\ F r_{\beta} & {[-]} & \text { Modified Froude number } \\ H_{\mathrm{sat}} & {[\mathrm{kJ} / \mathrm{kg}]} & \text { Latent heat } \\ H_{\mathrm{sh}} & {[\mathrm{kJ} / \mathrm{kg}]} & \text { Sensible heat } \\ g & {\left[\mathrm{~m} / \mathrm{s}^{2}\right]} & \text { Acceleration of gravity } \\ G r & {\left[\mathrm{~kg} / \mathrm{m}^{2} \mathrm{~s}\right]} & \text { Mass flus } \\ j & {[\mathrm{~m} / \mathrm{s}]} & \text { Superficial velocity } \\ L & {[\mathrm{~mm}]} & \text { Run up distance } \\ M & {[\mathrm{~kg} / \mathrm{s}]} & \text { Mass flow rate } \\ P & {[\mathrm{~Pa}]} & \text { Pressure } \\ Q & {[\mathrm{~kW}]} & \text { Capacity } \\ R_{c} & {[\mathrm{~m}]} & \text { Bending diameter } \\ v_{m} & {[\mathrm{~m} / \mathrm{s}]} & \text { Mean velocity } \\ x & {[-]} & \text { Quality } \\ \text { Greek } & & \\ \beta & {[-]} & \text { Bending Angle } \\ \rho & {\left[\mathrm{kg} / \mathrm{m}^{3}\right]} & \text { Density } \\ \theta & {\left[{ }^{\circ}\right]} & \text { Orientation angle } \\ \text { subscription } & & \\ 1,2 & & \text { Outlet of junction } \\ \mathrm{G} & & \text { Gas } \\ \text { in } & & \text { Inlet } \\ \mathrm{L} & & \text { Liquid }\end{array}$

\section{References}

[1] A. Aziz, A. Miyara and F. Sugino, "Distribution of two-phase flow in a distributor," J. Eng. Sci. Tech., vol. 7, no. 1, pp. 41-55, 2012.

[2] B. J. Azzopardi, A. Purvis and A. H. Govan, "Annular two-phase flow split at an impacting T," Int. J. Multiphase Flow. vol. 13 no. 5, pp. 605-614, 1987. 
[3] I. Yoshimura and A. Ishii, "Distribution Prediction for Distributor using Gas-liquid Flow Simulation with Liquid Film Modification," Trans. of the JSRAE, vol. 29, no. 3, pp. 331-342, 2012.

[4] M. Billiet, Y. Bastien, S. Lecompte, L. Marija, A. Kaya and M. Paepe, "The distribution of two-phase R32 over an impacting T-junction" presented at the 9th World Conference on Experimental Heat Transfer, Fluid Mechanics and Thermodynamics, Iguazu Falls, Brazil, 2017. [Online]. Available: http://hdl.handle.net/1854/LU-8526489

[5] Y. Taitel, D. Barnea and A. E. Dukler, "Modeling flow pattern transitions for steady upward gas-liquid flow in vertical tubes," AIChE J., vol. 26 no. 3, 345-354, 1980.

[6] G. C. Gardner and P. H. Neller, "Phase Distributions in Flow of an Air-Water Mixture round Bends and past Obstructions at the Wall of a 76-mm Bore Tube," in Proc. Inst. Mech. Eng. Conf. Proc., vol. 184, no. 3, pp. 93-101, 1969.

[7] G. B. Wallis, "One-dimensional two phase flow," McGraw-Hill, 1969. 